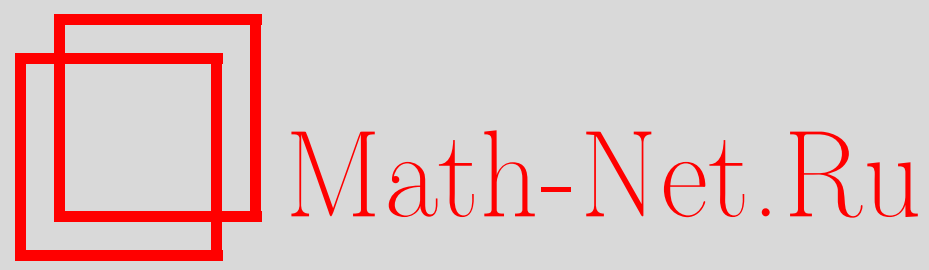

В. А. Кулагин, Об алгебраической независимости значений гипергеометрических функций одного класса, Матем. заметки, 1996, том 59, выпуск $3,402-414$

DOI: https://doi.org/10.4213/mzm1728

Использование Общероссийского математического портала MathNet.Ru подразумевает, что вы прочитали и согласны с пользовательским соглашением

http://www . mathnet.ru/rus/agreement

Параметры загрузки:

IP : 3.89 .197 .203

26 апреля 2023 г., 14:00:59

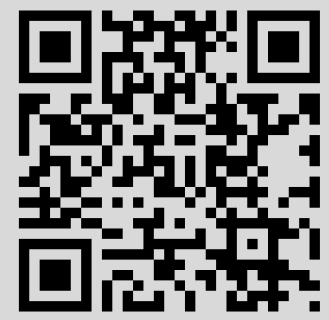




\section{ОБ АЛГЕБРАИЧЕСКОЙ НЕЗАВИСИМОСТИ ЗНАЧЕНИЙ ГИПЕРГЕОМЕТРИЧЕСКИХ ФУНКЦИЙ ОДНОГО КЛАССА}

\section{В.А. Кулагин}

Введение. Пусть $f_{1}(z), \ldots, f_{m}(z)$ - Е-функции, составляющие решение системы дифференциальных уравнений

$$
y_{i}^{\prime}=q_{i, 0}+\sum_{j=1}^{m} q_{i, j} y_{j}, \quad i=1, \ldots, m,
$$

$q_{i, j} \in \mathbb{C}(z), \alpha$-алгебраическое число, отличное от нуля и полюсов всех $q_{i, j}$.

В работе [1] А.Б. Шидловский доказал, что алгебраическая независимость над $\mathbb{Q}$ чисел $f_{1}(\alpha), \ldots, f_{m}(\alpha)$ эквивалентна алгебраической независимости над $\mathbb{C}(z)$ функций $f_{1}(z), \ldots, f_{m}(z)$. Для применения этой теоремы к конкретным функциям многими авторами были разработаны методы доказательства алгебраической независимости функций над $\mathbb{C}(z)$ (история вопроса освещена в монографии [2]).

В работах В.Х. Салихова [3], [4] и Ф. Бейкерса, В. Д. Браунвелла и Г. Хекмана [5] были созданы глубокие методы доказательства алгебраической независимости гипергеометрических функций и их последовательных производных. В их результатах на значения параметров гипергеометрических функций накладьвались некоторые ограничения. В том числе такие, что в символах, стояших в числителях и знаменателях коэффищиентов степенных рядов функций, не должны происходить существенные сокрашения ("невырожденные" случаи).

В работе [6], используя теорему А.Б. Шидловского, другим методом была доказана общая теорема об алгебраической независимости значений Е-функций, которая может быть применена к гипергеометрическим функциям в "вырожденных" случаях.

В настоящей статье, являющейся продолжением работы [6], эта теорема применяется к конкретным Е-функциям. 
Рассмотрим функцию

$$
\varphi_{k, m}(z)=1+\sum_{n=1}^{\infty} \frac{1}{\left(\lambda_{1}+1\right)_{n} \cdots\left(\lambda_{k}+1\right)_{n}\left(\mu_{1}+n\right) \cdots\left(\mu_{m}+n\right)}\left(\frac{z}{k}\right)^{n k},
$$

где $(\lambda)_{n}=\lambda(\lambda+1) \cdots(\lambda+n-1), \lambda_{i}, \mu_{j} \neq-1,-2, \ldots, i=1, \ldots, k$, $j=1, \ldots, m, k, m \in \mathbb{N}$.

Пусть $\{a\}=\left\{a_{1}, \ldots, a_{k}\right\},\{b\}=\left\{b_{1}, \ldots, b_{k}\right\}$, все $a_{i}, b_{i} \in \mathbb{C}$. В дальнейшем $\{a\} \equiv\{b\}(\bmod \mathbb{Z})$ означает, что существует перестановка $i_{1}, \ldots, i_{k}$ чисел $1, \ldots, k$ такая, что $\left(a_{j}-b_{i_{j}}\right) \in \mathbb{Z}, j=1, \ldots, k$.

Теорема 1. Пусть $k>2$ - простое иисло, $\alpha \neq 0$ - алгебраическое иисло, $\lambda_{i}, \mu_{j} \in \mathbb{Q}, \lambda_{i}, \mu_{j} \neq-1,-2, \ldots, 1 \leqslant i \leqslant k, 1 \leqslant j \leqslant m$.

Тогда для алгебраической независимости чисел

$$
\varphi_{k, m}(\alpha), \varphi_{k, m}^{\prime}(\alpha), \ldots, \varphi_{k, m}^{(k+m-1)}(\alpha)
$$

необходимо и достаточно, чтобы выполнялись условия

$$
\begin{gathered}
\left(\mu_{j}-\lambda_{i}\right) \notin \mathbb{N}, \quad 1 \leqslant i \leqslant k, \quad 1 \leqslant j \leqslant m ; \\
\left(\mu_{i}-\mu_{j}\right) \notin \mathbb{Z} \backslash\{0\}, \quad 1 \leqslant i, j \leqslant m ; \\
\left\{\lambda_{1}, \ldots, \lambda_{k}\right\} \not\left\{0, \frac{1}{k}, \ldots, \frac{k-1}{k}\right\}(\bmod \mathbb{Z}) .
\end{gathered}
$$

Для доказательства требуется следующая теорема.

Tеорема 2. Пусть $k \in \mathbb{N}, \mu_{j} \in \mathbb{Q},-\mu_{j} \notin \mathbb{N}, j=1, \ldots, m$,

$$
\left\{\lambda_{1}, \ldots, \lambda_{k}\right\} \equiv\left\{\lambda, \lambda+\frac{1}{k}, \ldots, \lambda+\frac{k-1}{k}\right\} \quad(\bmod \mathbb{Z}), \quad 0<\lambda<\frac{1}{k} .
$$

Тогда

1) если $k$ - четное число и выполняются условия (2), (3) и

$$
\frac{k}{2}\left(2 \lambda-\mu_{j}\right) \notin \mathbb{Z}, \quad 1 \leqslant j \leqslant m,
$$

то числа (1) алгебраически независимы,

2) если $k$ - нечетное число, то для алгебраической независимости чисел (1) необходимо и достаточно, чтобы выполнялись только условия (2) и (3).

Формулировки теорем опубликованы в [7]. Достаточность условий теоремы 1 при $k=1,2$ была установлена А.Б. Шидловским [8]. Доказательство теоремы 1 использует результат В.Х. Салихова [3] (случай $m=0$ ). 
1. Вспомогательные утверждения. Рассмотрим дифференциальное уравнение с комплексными коэффициентами

$$
y^{\prime}+\left(\frac{a}{z}+b\right) y+\frac{c}{z}=h z^{n}+g z^{m},
$$

$a \notin \mathbb{N}, \quad b \neq 0, \quad c \neq 0 ; n, m \in \mathbb{Z} \backslash\{-1\}, \quad n \geqslant m$.

Введем обозначение $P(z)=h z^{n}+g z^{m}$. Если $h \neq 0$ и $g \neq 0$, то положим $N=\max \{-1, n\}, M=\min \{0, m+1\}$.

ЛЕмма 1. Если уравнение (6) имеет частное решение $v \in \mathbb{C}(z)$, то $P(z) \not \equiv 0$, а $v$ представима в виде суммы

$$
v=\sum_{i=M}^{N} x_{i} z^{i}, \quad x_{i} \in \mathbb{C}, \quad x_{N} \neq 0, \quad x_{M} \neq 0 .
$$

ДОКАЗАТЕЛЬСТВО. В конечной части плоскости особой точкой функции $v$ может быть только точка $z=0$. В ее окрестности $v$ представима в виде суммы $v=x_{r} z^{r}+\cdots+x_{l} z^{l}, l, r \in \mathbb{Z}, x_{r} \neq 0, x_{l} \neq 0$. Подставляя $v$ в (6) и сравнивая коэффициенты при $z^{l}$, а затем при $z^{r-1}$, получим утверждение леммы.

СлеДСТВИЕ. Если $a \notin \mathbb{N}, b \neq 0 \quad u \quad c \neq 0$, то уравнение $y^{\prime}+(a / z+b) y+c / z=0$ не имеет решений из $\mathbb{C}(z)$.

ЛЕмма 2. Если $a \notin \mathbb{Z}$ или $b \neq 0$, то уравнение $y^{\prime}+(a / z+b) y=0$ не имеет решений из $\mathbb{C}(z)$, кроме $y \equiv 0$.

Утверждение очевидно.

Пусть $v \in \mathbb{C}(z)$ есть решение (6) и имеет место (7).

ОПРедЕЛЕНИЕ. Систему линейных уравнений относительно $x_{N}, \ldots, x_{M}$, полученную в результате сравнения в уравнении (6) коэффициентов при всех степенях $z$ после подстановки в него (7), будем назьвать определяющей системой уравнений для $v$.

В дальнейшем, если две системы аналитических в некоторой области функций $F_{1}, \ldots, F_{n}$ и $\Phi_{1}, \ldots, \Phi_{n}$ алгебраически эквивалентны над $\mathbb{C}(z)$, то это будем обозначать $\left(F_{1}, \ldots, F_{n}\right) \sim\left(\Phi_{1}, \ldots, \Phi_{n}\right)$. (Определение алгебраической эквивалентности см. в $[9$, с. 264].)

Лемма 3. Пусть функиии $u_{1}(z), \ldots, u_{n}(z)$ линейно над $\mathbb{C}(z)$ выражаются через функиии $v_{1}(z), \ldots, v_{n}(z)$

$$
u_{i}(z)=q_{i, 0}+\sum_{j=1}^{n} q_{i, j} v_{j}(z), \quad i=1, \ldots, n,
$$

u определитель $\left|q_{i, j}\right| \neq 0,1 \leqslant i, j \leqslant n$.

Тогда $\left(u_{1}(z), \ldots, u_{n}(z)\right) \sim\left(v_{1}(z), \ldots, v_{n}(z)\right)$. 
ДокАЗАТЕЛЬСТво имеется в [2, с. 239].

Лемма 4. Пусть $\left(\mu_{j}-\lambda_{i}\right) \notin \mathbb{N}, l_{i} \in \mathbb{N} \cup\{0\}, j=1, \ldots, m, i=1, \ldots, k$, а функиия $F$ получена из $\varphi_{k, m}$ заменой параметров $\lambda_{i} \rightarrow \lambda_{i}+l_{i}$, $i=1, \ldots, k$.

Тогда $\left(F, F^{\prime}, \ldots, F^{(m+k-1)}\right) \sim\left(\varphi_{k, m}, \varphi_{k, m}^{\prime}, \ldots, \varphi_{k, m}^{(m+k-1)}\right)$.

Эта лемма при $m=0$ доказана В.Х. Салиховым [3] (доказательство имеется также в $[2$, с. 330$])$.

ДокаЗАТЕЛЬСТво. Обозначим $\delta=z \frac{d}{d z}$. Пусть для некоторого $s$, $1 \leqslant s \leqslant k, l_{s} \geqslant 1$, и функция $f$ получена из $F$ заменой $\lambda_{s} \rightarrow \lambda_{s}-1$. Легко проверить, что

$$
\left(\delta+k\left(\lambda_{s}+l_{s}\right)\right)(F)=k\left(\lambda_{s}+l_{s}\right) f
$$

и $F$ удовлетворяет дифференщиальному уравнению

$$
\mathscr{L}(y)=k^{m} z^{k}
$$

где дифференциальньй оператор $\mathscr{L}$ имеет вид

$$
\mathscr{L}=\left(\prod_{i=1}^{k}\left(\delta+k\left(\lambda_{i}+l_{i}\right)\right)-z^{k}\right) \prod_{j=1}^{m}\left(\delta+k \mu_{j}\right)
$$

Из (8) и (9) получаем, что функции

$$
f, f^{\prime}, \ldots, f^{(m+k-1)}
$$

линейно над $\mathbb{C}(z)$ выражаются через функции

$$
F, F^{\prime}, \ldots, F^{(m+k-1)}
$$

Докажем теперь, что функции (11) таким же образом выражаются через (10). С помощью равенств

$$
\delta+k \mu_{j}=\delta+k\left(\lambda_{s}+l_{s}\right)+k\left(\mu_{j}-\lambda_{s}-l_{s}\right)
$$

оператор $\mathscr{L}$ представим в виде

$$
\mathscr{L}=\mathscr{L}_{1}\left(\delta+k\left(\lambda_{s}+l_{s}\right)\right)+\gamma,
$$


где

$$
\begin{gathered}
\mathscr{L}_{1}=\delta^{k+m-1}+a_{k+m-2} \delta^{k+m-2}+\cdots+a_{0}, \quad a_{i} \in \mathbb{C}[z], \\
\gamma=z^{k} k^{m} \prod_{j=1}^{m}\left(\lambda_{s}+l_{s}-\mu_{j}\right) .
\end{gathered}
$$

По условию леммы $\left(\mu_{i}-\lambda_{j}\right) \notin \mathbb{N}$, и поэтому $\gamma \neq 0$. Из (12) и (8) получим $\mathscr{L}(F)=\mathscr{L}_{1}\left(\delta+k\left(\lambda_{s}+l_{s}\right)(F)+\gamma F=k\left(\lambda_{s}+l_{s}\right) \mathscr{L}_{1}(f)+\gamma F\right.$, а так как $F(z)$ удовлетворяет $(9)$, то

$$
F(z)=\frac{k^{m} z^{k}}{\gamma}+k \frac{\left(\lambda_{s}+l_{s}\right)}{\gamma} \mathscr{L}_{1}(f)
$$

Функция $f(z)$, аналогично $F(z)$, удовлетворяет уравнению

$$
\mathscr{L}_{2}(y)=k^{m} z^{k}
$$

где оператор $\mathscr{L}_{2}$ получается из $\mathscr{L}_{1}$ заменой $\lambda_{s} \rightarrow \lambda_{s}-1$. Из (13), (12) и (14) получим, что функции (11) линейно над $\mathbb{C}(z)$ выражаются через функции (10). Следовательно, эти совокупности функций алгебраически эквивалентны над $\mathbb{C}(z)$.

После нужного количества повторений указанных рассуждений получим утверждение леммы.

2. Доказательство теоремы 2. Можно считать, ввиду леммы 4, что $\lambda_{i}=\lambda+(i-1) / k, i=1, \ldots, k$. Обозначим $\Lambda=\lambda k+k-1$,

$$
\psi_{\Lambda}(z)=1+\sum_{n=1}^{\infty} \frac{z^{n}}{(\Lambda+1)_{n}}
$$

$\Phi$ ункция

$$
\varphi_{k}(z)=1+\sum_{n=1}^{\infty} \frac{1}{\left(\lambda_{1}+1\right)_{n} \cdots\left(\lambda_{k}+1\right)_{n}}\left(\frac{z}{k}\right)^{n k}
$$

связана с $\psi_{\Lambda}(z)$ следующим образом:

$$
\varphi_{k}(z)=\frac{1}{k} \sum_{i=1}^{k} \psi_{\Lambda}\left(\xi^{i} z\right)
$$


где $\xi$ - первообразньй корень степени $k$ из 1 . Доказательство разложения $\varphi_{k}(z)$ в сумму $(15)$ имеется в $[2$, c. 340$]$. В дальнейшем для удобства считаем $\xi=\exp \{2 \pi i / k\}$. Рассмотрим функции

$$
\left.\begin{array}{rl}
\omega_{j}(z) & =\psi_{\Lambda}\left(\xi^{j} z\right), \quad j=1, \ldots, k \\
\omega_{k+i}(z) & =\sum_{n=1}^{\infty} \frac{1}{\prod_{s=1}^{k}\left(\lambda+1+\frac{s-1}{k}\right)_{n}\left(\mu_{1}+n\right) \cdots\left(\mu_{i}+n\right)}\left(\frac{z}{k}\right)^{n k}, \\
i & =1, \ldots, m,
\end{array}\right\}
$$

удовлетворяющие системе дифференциальных уравнений

$$
\left.\begin{array}{rlrl}
y_{j}^{\prime} & =\left(-\frac{\Lambda}{z}+\xi^{j}\right) y_{j}+\frac{\Lambda}{z}, & j=1, \ldots, k, \\
y_{k+1}^{\prime} & =-\frac{k}{z} \mu_{1} y_{k+1}+\frac{1}{z}\left(y_{1}+\cdots+y_{k}\right), & & \\
y_{k+i}^{\prime} & =-\frac{k}{z} \mu_{i} y_{k+i}+\frac{k}{z} y_{k+i-1}, & i & =2, \ldots, m .
\end{array}\right\}
$$

Покажем, что

$$
\left(\varphi_{k, m}, \varphi_{k, m}^{\prime}, \ldots, \varphi_{k, m}^{(k+m-1}\right) \sim\left(\omega_{1}, \ldots, \omega_{k+m}\right) .
$$

Исходя из равенства $\varphi_{k, m}(z)=\omega_{k+m}(z)$ и пользуясь уравнениями системы (17), получим: если $i=1, \ldots, m-1$, то

$$
\varphi_{k, m}^{(i)}=\left(\frac{k}{z}\right)^{i} \omega_{k+m-i}+\sum_{j=0}^{i-1} a_{i, j} \omega_{k+m-j}
$$

а если $i=m$, то

$$
\varphi_{k, m}^{(m)}=\frac{1}{z}\left(\frac{k}{z}\right)^{m-1} \sum_{j=1}^{m} \omega_{j}+\sum_{j=0}^{m-1} a_{m, j} \omega_{k+m-j}, \quad a_{i, j} \in \mathbb{C}(z) .
$$

Индукцией по $i$ легко доказать, что функции $\varphi_{k, m}^{(i)}, i=m+1, \ldots, m+k-1$, можно представить в виде линейной формы

$$
\begin{gathered}
\varphi_{k, m}^{(i)}=\sum_{j=1}^{k}\left(\sum_{s=0}^{i-m} b_{i, s} \xi^{j s}\right) \omega_{j}+\sum_{j=0}^{m-1} a_{i, j} \omega_{k+m-j} \\
b_{i, s}, a_{i, j} \in \mathbb{C}(z), \quad b_{i, i-m} \neq 0 .
\end{gathered}
$$


Таким образом, $\varphi_{k, m}^{(i)}, i=0,1, \ldots, m+k-1$, линейно над $\mathbb{C}(z)$ выражаются через функции (16), и определитель матрицы перехода равен $\prod_{1 \leqslant i<j \leqslant k}\left(\xi^{i}-\xi^{j}\right) \prod_{s=0}^{k-1} b_{m+s, s} \neq 0$. По лемме 3 эквивалентность (18) доказана. Поэтому для доказательства теоремы достаточно установить ее утверждение для чисел $\omega_{1}(\alpha), \ldots, \omega_{m}(\alpha)$.

1) Пусть $k$ - четное число, $k=2 l$. Докажем, что функции (16) удовлетворяют условиям А), В), С) и D) теоремы работы [1]. Для $n=k+1, \ldots, k+m$ положим $\beta=2 \Lambda-k \mu_{n-k}$.

А) Функции $\omega_{1}(z), \ldots, \omega_{k}(z)$ алгебраически независимы над $\mathbb{C}(z)$. Это доказано в [8].

В) Дифференциальное уравнение $y_{i}^{\prime}=\left(\xi^{i}-\Lambda / z\right) y_{i}+\Lambda / z, i=1, \ldots, k$, не имеет решений из $\mathbb{C}(z)$ (следствие из леммы 1$)$.

С) Дифференциальное уравнение $y^{\prime}=\left(\xi^{i}-\xi^{j}\right) y, i \neq j, 1 \leqslant i, j \leqslant k$, имеет только тривиальное решение из $\mathbb{C}(z)$. Очевидно.

D) Докажем, что для $n=k+1, \ldots, m$ и любого решения $v_{i, j} \in \mathbb{C}(z)$ системы дифференциальных уравнений

$$
y_{i, j}^{\prime}=\left(\frac{\beta}{z}-\xi^{i}-\xi^{j}\right) y_{i, j}, \quad 1 \leqslant i, j \leqslant k,
$$

такого, что $v_{i, j}=v_{j, i}$, и любого набора натуральных $t_{1}, \ldots, t_{k}$ система дифференциальных уравнений

$$
Y^{\prime}+Y\left(A_{n-1}-q_{n, n} E_{n-1}\right)+Q_{n-1}=V_{n-1},
$$

где $Y=\left(y_{1}, \ldots, y_{n-1}\right), A_{n-1}=\left(q_{i, j}\right)$ - матрица коэффициентов при $y_{1}, \ldots, y_{n-1}$ первых $n-1$ уравнений системы $(17), E_{n-1}-$ единичная матрища порядка $n-1, V_{n-1}=\left(u_{1}, \ldots, u_{k}, 0, \ldots, 0\right)$, $\operatorname{dim} V_{n-1}=n-1, \quad u_{i}=\frac{\Lambda}{z} \sum_{j=1}^{k} t_{j} v_{i, j}, \quad Q_{n-1}=\left(q_{n, 1}, \ldots, q_{n, n-1}\right)$, не имеет решений, состоящих из рациональных функций.

Из леммы 2 следует, что решение $v_{i, j} \in \mathbb{C}(z)$ системы (19) имеет вид

$$
v_{i, j}= \begin{cases}0, & \text { если } \beta \notin \mathbb{Z} \text { или }|i-j| \neq l, \\ c_{i, j} z^{\beta}, & \text { если } \beta \in \mathbb{Z} \text { и } \quad|i-j|=l, \quad c_{i, j} \in \mathbb{C} .\end{cases}
$$

Рассмотрим функции $(21)$, для которых $c_{i, j}=c_{j, i}(1 \leqslant i, j \leqslant k)$. Тогда в (20) $V_{n-1}$ имеет вид: $V_{n-1}=z^{\beta-1}\left(a_{1}, \ldots, a_{k}, 0, \ldots, 0\right)$, где все $a_{i}=0$, если $\beta \notin \mathbb{Z}$, а при $\beta \in \mathbb{Z}$

$$
a_{i}= \begin{cases}\Lambda c_{i, i+l} t_{i+l}, & 1 \leqslant i \leqslant l \\ \Lambda c_{i, i-l} t_{i-l}, & l<i \leqslant k .\end{cases}
$$


Для этого заметим, что при $\beta \in \mathbb{Z}$ для каждого $i, 1 \leqslant i \leqslant k$, сушествует только одно $j, 1 \leqslant j \leqslant k$, для которого $|i-j|=l$, а именно: $j=i+l$, если $i \leqslant l$, и $j=i-l$, если $i>l$.

Из предположения $c_{i, j}=c_{j, i}, 1 \leqslant i, j \leqslant k$, следует

$$
a_{i+l} t_{i+l}=a_{i} t_{i}
$$

Докажем теперь, что для $n=k+1, \ldots, k+m$ соответствующая система (20) не имеет решений, состоящих из рациональных функций. Рассмотрим следующие случаи.

1.1) Пусть $n=k+1$. Тогда $\beta=2 \Lambda-k \mu_{1}$ и (20) имеет вид

$$
y_{i}^{\prime}+\left(\xi^{i}+\frac{1}{z}\left(k \mu_{1}-\Lambda\right)\right) y_{i}+\frac{1}{z}=a_{i} z^{\beta-1}, \quad i=1, \ldots, k .
$$

Из условия (2) следует, что при любом $i, 0 \leqslant i \leqslant k-1$,

$$
\left(\mu_{1}-\lambda-\frac{i}{k}\right) \notin \mathbb{N} \text {, т.е. } k \mu_{1}-\Lambda=\left(k\left(\mu_{1}-\lambda\right)-k+1\right) \notin \mathbb{N} \text {. }
$$

Если найдется $a_{i}=0$, то по следствию леммы $1, i$-е уравнение системы (24) не имеет решений из $\mathbb{C}(z)$. Предположим, что $\beta \in \mathbb{Z}$ и все $a_{i} \neq 0$. Возможны три подслучая:

a) Пусть $\beta=0$. Тогда $2 \Lambda-k \mu_{1}=2 \lambda k+2 k-2-k \mu_{1}=0$, или $\frac{k}{2}\left(2 \lambda-\mu_{1}\right)=1-k$, что противоречит условию (5). Следовательно, этот случай невозможен.

в) Пусть $\beta>0$ и (24) имеет некоторое решение $v_{1}, \ldots, v_{k} \in \mathbb{C}(z)$. По лемме $1 v_{j}$ разложима в сумму $v_{j}=x_{0, j}+x_{1, j} z+\cdots+x_{\beta-1, j} z^{\beta-1}$ и определяющая система линейных уравнений для $v_{j}$ имеет вид

$$
\left.\begin{array}{c}
\left(\mu_{1}-\mu_{2}\right) x_{0, j}=1, \\
\left(\Lambda-k \mu_{1}-i\right) x_{i, j}=\xi^{j} x_{i-1, j}, \quad i=1, \ldots, \beta-1, \\
a_{j}=\xi^{i} x_{\beta-1, j} .
\end{array}\right\}
$$

Так как $\left(\Lambda-k \mu_{1}\right) \notin \mathbb{Z}$, то $x_{i, j} \neq 0, i=0,1, \ldots, \beta-1$. Перемножая эти равенства и сокращая результат на $x_{0, j} \cdots x_{\beta-1, j}$, получим

$$
a_{j}\left(\mu_{1}-\mu_{2}\right) \prod_{i=0}^{\beta-1}\left(\Lambda-k \mu_{1}-i\right)=\xi^{j \beta} .
$$


Разделим левые и правые части (26) при $j=k$ и $j=l$. Учитьвая (23), получим $t_{k}=t_{l} \exp (\beta \pi i)$. Следовательно, $\beta$-четное число. Но $\beta=2 \Lambda-k \mu_{1}$ $=2\left(l\left(2 \Lambda-\mu_{1}\right)+k-1\right)$ и поэтому $l\left(2 \Lambda-\mu_{1}\right) \in \mathbb{Z}$, что противоречит условию (5).

c) $\beta<0$. Этот случай аналогичен предыдущему.

$1.2)$ Пусть $n=k+2, \beta=2 \Lambda-k \mu_{2}$. Положим $\sigma=k\left(\mu_{1}-\mu_{2}\right)$. В данном случае система (20) имеет вид

$$
\begin{gathered}
y_{i}^{\prime}+\left(\xi^{i}+\frac{1}{z}\left(k \mu_{2}-\Lambda\right)\right) y_{i}+\frac{1}{z} y_{k+1}=a_{i} z^{\beta-1}, \quad i=1, \ldots, k, \\
y_{k+1}^{\prime}-\frac{\sigma}{z} y_{k+1}+\frac{k}{z}=0 .
\end{gathered}
$$

Если $\sigma=0$, то последнее уравнение, очевидно, не имеет решений из $\mathbb{C}(z)$ и утверж дение доказано. Предположим, что $\sigma \neq 0$. Интегрируя это уравнение, найдем $y_{k+1}=k / \sigma+c_{0} z^{\sigma}, c_{0} \in \mathbb{C}$. Рассмотрим теперь подсистему из $k$ первых уравнений $(27)$

$$
\begin{gathered}
y_{i}^{\prime}+\left(\xi^{i}+\frac{1}{z}\left(k \mu_{2}-\Lambda\right)\right) y_{i}+\frac{k}{\sigma z}+c_{0} z^{\sigma-1}=a_{i} z^{\beta-1}, \\
i=1, \ldots, k,
\end{gathered}
$$

и докажем, что она не имеет решений, состоящих из рациональных функций. Если $c_{0}=0$, то это доказьвается аналогично 1.1).

Пусть $c_{0} \neq 0, \sigma \in \mathbb{Z}$. Допустим, что утверждение неверно: существуют $f_{1}, \ldots, f_{k} \in \mathbb{C}(z)$, удовлетворяющие системе (28). Рассмотрим два подслучая.

a) $a_{i}=0, i=1, \ldots, l$. Тогда из (23) следует, что $a_{i}=0$ при $i=l+1$, $\ldots, k$. По лемме 2 каждую функцию $f_{j}$ можно разложить в сумму

$$
f_{j}=\sum_{i=0}^{\sigma-1} x_{i, j} z^{i}, \quad \text { если } \sigma \in \mathbb{N},
$$

И

$$
f_{j}=\sum_{i=\sigma}^{-1} x_{i, j} z^{i}, \quad \text { если }-\sigma \in \mathbb{N}, x_{i, j} \in \mathbb{C} .
$$

Если имеет место (29), то определяющая система для $f_{j}$ имеет вид

$$
\left.\begin{array}{c}
\left(\mu_{1}-\mu_{2}\right)\left(\Lambda-k \mu_{2}\right) x_{0, j}=1, \\
\left(\Lambda-k \mu_{2}-i\right) x_{i, j}=x_{i-1, j} \xi^{i}, \quad i=1, \ldots, \sigma-1, \\
c_{0}=-x_{\sigma-1, j} .
\end{array}\right\}
$$


Аналогично тому, как из (25) было получено (26), из (31) при $\mu_{1} \neq \mu_{2}$ получим равенство

$$
c_{0}\left(\mu_{2}-\mu_{1}\right) \prod_{i=0}^{\sigma-1}\left(\Lambda-k \mu_{2}-i\right)=\xi^{\sigma j},
$$

в котором левая часть не зависит от $j$. Поэтому при $j=1$ и $j=k$ она должна иметь одно и то же значение, т.е. $\xi^{\sigma}=\xi^{\sigma k}=\exp (2 \pi i \sigma)=1$. Так как $\xi^{\sigma}=\exp \left(2 \pi i\left(\mu_{1}-\mu_{2}\right)\right)$, то отсюда следует, что $\left(\mu_{1}-\mu_{2}\right) \in \mathbb{Z}$, вопреки условию (3).

Случай, когда имеет место (30), рассматривается аналогично.

b) Пусть для некоторого $r, 1 \leqslant r \leqslant l, a_{r} \neq 0$. Тогда из (23) следует, что $a_{r+l} \neq 0$. Рассмотрим $r$-е и $(r+l)$-е уравнения системы $(27)$ и докажем, что они не могут одновременно иметь решения из $\mathbb{C}(z)$. Допустим противное: пусть $f_{r}, f_{r+l} \in \mathbb{C}(z)$ и удовлетворяют рассматриваемым уравнениям. Предположим, что $\sigma>1$ и $\beta>\sigma$ (остальные возможные случаи рассматриваются аналогично). Пусть $j$ принимает значение $r$ или $r+l$. По лемме 1

$$
f_{j}=\sum_{i=0}^{\beta-1} x_{i, j} z^{i}, \quad x_{i, j} \in \mathbb{C},
$$

и определяюшая система для $f_{j}$ имеет вид

$$
\left.\left.\begin{array}{c}
\left(\Lambda-k \mu_{2}\right)\left(\mu_{1}-\mu_{2}\right) x_{0, j}=1, \\
\left(\Lambda-k \mu_{2}-i\right) x_{i, j}=x_{i-1, j} \xi^{j}, \quad i=1, \ldots, \sigma-1,
\end{array}\right\} \begin{array}{c}
\left(\Lambda-k \mu_{1}\right) x_{\sigma, j}=x_{\sigma-1, j} \xi^{j}+c_{0}, \\
\left(\Lambda-k \mu_{2}-i\right) x_{i, j}=x_{i-1, j} \xi^{j}, \quad i=\sigma+1, \ldots, \beta-1, \\
a_{j}=x_{\beta-1, j} \xi^{j} .
\end{array}\right\}
$$

Из (32) и (34) соответственно получим равенства

$$
\begin{gathered}
\left(\mu_{1}-\mu_{2}\right) x_{\sigma-1, j} \prod_{i=0}^{\sigma-1}\left(\Lambda-k \mu_{2}-i\right)=\xi^{j(\sigma-1)}, \\
a_{j} \prod_{i=\sigma+1}^{\beta-1}\left(\Lambda-k \mu_{2}-i\right)=\xi^{j(\beta-\sigma)} x_{\sigma, j}, \quad x_{\sigma, j} \neq 0 .
\end{gathered}
$$

Положив в (35) $j=r$ и $j=r+l$, и разделив одноименные части полученных равенств, найдем

$$
x_{\sigma-1, r+l}=x_{\sigma-1, r} e^{\pi i(\sigma-1)} .
$$


Точно так же из (36) с помощью (23) получим

$$
t_{r+l} x_{\sigma, r+l}=t_{r} x_{\sigma, r} e^{\beta \pi i} .
$$

Из (5) следует, что $\beta$ - нечетное число, поскольку $\beta=2 \Lambda-k \mu_{2}=$ $k\left(2 \lambda-\mu_{2}\right)+2 k-2$. Аналогично, $\left(2 \Lambda-k \mu_{1}\right)$ - нечетное число, а тогда их разность, равная $k\left(\mu_{1}-\mu_{2}\right)=\sigma-$ четное число. Поэтому (37) и (38) соответственно примут вид

$$
\begin{aligned}
x_{\sigma-1, r+l} & =-x_{\sigma-1, r} \\
t_{r+l} x_{\sigma, r+l} & =-t_{r} x_{\sigma, r} .
\end{aligned}
$$

Рассмотрим уравнение (33) при $j=r$ и $j=r+l$ :

$$
\begin{aligned}
\left(\Lambda-k \mu_{1}\right) x_{\sigma, r} & =\xi^{r} x_{\sigma-1, r}+c_{0}, \\
\left(\Lambda-k \mu_{1}\right) x_{\sigma, r+l} & =\xi^{r+l} x_{\sigma-1, r+l}+c_{0}=-\xi^{r} x_{\sigma-1, r+l}+c_{0} .
\end{aligned}
$$

Из (39), (40) и (42) получим

$$
\left(k \mu_{1}-\Lambda\right) t_{r} x_{\sigma, r}=t_{r+l}\left(\xi^{r} x_{\sigma-1, r}+c_{0}\right) .
$$

Так как $t_{r}, t_{r+l} \in \mathbb{N}$, то из (41) и (43) следует $x_{\sigma, r}=0$, что по предположению невозможно. Полученное противоречие доказывает утверждение при $n=k+2$.

1.3) Пусть $k+2<n \leqslant k+m$. Тогда соответствуюшая система (20) имеет вид (положим $\beta=2 \Lambda-k \mu_{n-k}, \sigma=k\left(\mu_{n-k-1}-\mu_{n-k}\right)$ )

$$
\left.\begin{array}{ll}
y_{i}^{\prime}+\left(\xi^{i}+\frac{1}{z}\left(k \mu_{n-k}-\Lambda\right)\right) y_{i}+\frac{1}{z} y_{k+1}=a_{i} z^{\beta-1}, & i=1, \ldots, k, \\
y_{i}^{\prime}+\frac{k}{z}\left(\mu_{n-k}-\mu_{i-k}\right) y_{i}+\frac{k}{z} y_{k+1}=0, & i=k+1, \ldots, n-2, \\
y_{n-1}^{\prime}-\frac{\sigma}{z} y_{n-1}+\frac{k}{z}=0 . &
\end{array}\right\}
$$

Если $\sigma=0$, то последнее уравнение не имеет решений из $\mathbb{C}(z)$ и утверждение доказано. Пусть $\sigma \neq 0$. Тогда интегрируя это уравнение, получим $y_{n-1}=\frac{k}{\sigma}+c_{n-1} z^{\sigma}, c_{n-1} \in \mathbb{C}$. Из предыдущих уравнений найдем

$$
y_{i}=\prod_{j=i}^{n-1}\left(\mu_{j-k}-\mu_{n-k}\right)^{-1}+c_{i} z^{\sigma}, \quad c_{i} \in \mathbb{C}, \quad i=k+1, \ldots, n-2 .
$$


Поэтому подсистема из первых $k$ уравнений будет иметь вид, аналогичный системе (28), и, следовательно, также не имеет решений, состояших из рациональных функций. Утверждение 1) теоремы доказано.

2 ) Пусть $k$-нечетное число. Тогда по лемме 2 система (19) имеет только тривиальное решение из $\mathbb{C}(z)$. Действительно, для любых $i, j, 1 \leqslant i, j \leqslant k$, равенство $\xi^{i}+\xi^{j}=0$ выполняться не может. Поэтому для каждого $n$, $k+1 \leqslant n \leqslant k+m$, в соответствуюшей системе (20) вектор $V_{n-1} \equiv 0$. Заметим, что при доказательстве утверждения 1) теоремы для четных значений $k$ в тех случаях, когда все $a_{i}=0$, т.е. когда $V_{n-1} \equiv 0$, использовались только условия (2) и (3), а четность $k$ и условие (5) не использовались. Поэтому (2) и (3) при нечетном $k$ являются достаточньми условиями.

Докажем теперь необходимость этих условий. Допустим, что (2) не выполняется: пусть для некоторых $r, s, r \geqslant 1, s \geqslant 1, \mu_{s}-\lambda_{r}=l \in \mathbb{N}$. Можно считать, что $s=1$. Докажем, что функции $\omega_{k+1}, \omega_{k+1}^{\prime}, \ldots, \omega_{k+1}^{(k)}$ алгебраически зависимы над $\mathbb{C}(z)$. Рассмотрим функцию

$$
f=f(z)=\sum_{n=1}^{\infty} \frac{1}{\left(\lambda_{1}+1\right)_{n} \cdots\left(\lambda_{r}+l\right)_{n} \cdots\left(\lambda_{k}+1\right)_{n}\left(\mu_{1}+n\right)}\left(\frac{z}{k}\right)^{n k},
$$

которая получается из $\omega_{k+1}$ заменой $\lambda_{r} \rightarrow \lambda_{r}+l-1$. По лемме 4 $\left(\omega_{k+1}, \omega_{k+1}^{\prime}, \ldots, \omega_{k+1}^{(k)}\right) \sim\left(f, f^{\prime}, \ldots, f^{(k)}\right)$. Так как $\left(\mu_{1}+n\right)\left(\lambda_{r}+l\right)_{n}=$ $\left(\lambda_{r}+l\right)\left(\lambda_{r}+l+1\right)_{n}$, то

$$
f(z)=\frac{1}{\left(\lambda_{r}+l\right)} \sum_{n=1}^{\infty} \frac{1}{\left(\lambda_{1}+1\right)_{n} \cdots\left(\lambda_{r}+l+1\right)_{n} \cdots\left(\lambda_{k}+1\right)_{n}}\left(\frac{z}{k}\right)^{n k} .
$$

Но тогда $f(z)$ удовлетворяет дифференциальному уравнению $k$-о порядка и, следовательно, $f(z), f^{\prime}(z), \ldots, f^{(k)}(z)$ алгебраически зависимы над $\mathbb{C}(z)$.

Докажем теперь необходимость условия (3). Допустим, что оно не выполняется: например, $\mu_{2}-\mu_{1}=l \in \mathbb{N}$. Докажем, что $\omega_{1}, \ldots, \omega_{k+2}$ алгебраически зависимы над $\mathbb{C}(z)$. Обозначим

$$
v=v(z)=\sum_{n=1}^{\infty} \frac{1}{\left(\lambda_{1}+1\right)_{n} \cdots\left(\lambda_{k}+1\right)_{n}\left(\mu_{2}+n\right)}\left(\frac{z}{k}\right)^{n k} .
$$

Тогда $l \omega_{k+2}=\omega_{k+1}-v$, и поэтому $\left(\omega_{1}, \ldots, \omega_{k+2}\right) \sim\left(v, \omega_{1}, \ldots, \omega_{k+1}\right)$. Легко проверить равенство

$$
\begin{aligned}
& \omega_{k+1}=\sum_{n=1}^{l} \frac{1}{\prod_{i=1}^{k}\left(\lambda_{i}+1\right)_{n}\left(\mu_{1}+n\right)}\left(\frac{z}{k}\right)^{n k} \\
& +\prod_{i=1}^{k}\left(\lambda_{i}+1\right)_{l}^{-1}\left(\frac{z}{k}\right)^{k l} \sum_{n=1}^{\infty} \frac{1}{\left(\lambda_{1}+1+l\right)_{n} \cdots\left(\lambda_{k}+1+l\right)_{n}\left(\mu_{2}+n\right)}\left(\frac{z}{k}\right)^{n k},
\end{aligned}
$$


из которого по лемме 4 следует $\left(\omega_{k+1}, \omega_{k+1}^{\prime}, \ldots, \omega_{k+1}^{(k)}\right) \sim\left(v, v^{\prime}, \ldots, v^{(k)}\right)$. Ранее было установлено (см. (18)), что $\left(\omega_{k+1}, \omega_{k+1}^{\prime}, \ldots, \omega_{k+1}^{(k)}\right) \sim$ $\left(\omega_{1}, \ldots, \omega_{k+1}\right)$ и $\left(v, v^{\prime}, \ldots, v^{(k)}\right) \sim\left(v, \omega_{1}, \ldots, \omega_{k}\right) . \quad$ Следовательно, $\left(\omega_{1}, \ldots, \omega_{k+1}\right) \sim\left(v, \omega_{1}, \ldots, \omega_{k}\right)$. Таким образом, $\omega_{k+1}$ алгебраически над $\mathbb{C}(z)$ зависит от $v, \omega_{1}, \ldots, \omega_{k}$, и тем самым утверждение теоремы доказано.

3. Доказательство теоремы 1. Рассмотрим два случая.

1. Существует $\lambda \in \mathbb{Q}, 0<\lambda<1 / k$, такое, что

$$
\left\{\lambda_{1}, \ldots, \lambda_{k}\right\} \equiv\left\{\lambda, \lambda+\frac{1}{k}, \ldots, \lambda+\frac{k-1}{k}\right\} \quad(\bmod \mathbb{Z}) .
$$

Тогда по теореме 2 утверждение теоремы доказано.

2. Предположим, что для любого $\lambda \in \mathbb{Q}(44)$ не выполняется. В этом случае необходимость условий теоремы доказывается так же, как в теореме 2 , а достаточность установлена в работе [10].

Марийский государственньй университет

Поступило 16.06 .93

\section{СПИСОК ЦИТИРОВАННОЙ ЛИТЕРАТУРЫ}

[1] Шидловский А. Б. О критерии алгебраической независимости значений одного класса целых функций // Изв. АН СССР. Сер. матем. 1959. Т. 23. № 1. C. $35-66$.

[2] Шидловский А. Б. Трансцендентные числа. М.: Наука, 1987.

[3] Салихов В. Х. Формальные решения линейных дифференциальных уравнений и их применение в теории трансцендентных чисел // Тр. ММО. 1988. Т. 51. C. 223-256.

[4] Салихов В. Х. Об алгебраической независимости значений гипергеометрических Е-функций // Докл. АН СССР. 1989. Т. 307. № 2. С. 284-287.

[5] Beukers F., Brownawell W. D., Heckman G. Siegel normality // Ann. Math. 1988. V. 127. P. 279-308.

[6] Кулагин В. А. Об алгебраической независимости значений одного подкласса целых функций // Матем. заметки. 1992. Т. 51. №6. С. 46-51.

[7] Кулагин В.А. Об алгебраической независимости значений некоторых гипергеометрических функций // УМН. 1987. Т. 42. № 2(254). С. 233-234.

[8] Шидловский А. Б. О трансцендентности и алгебраической независимости значений некоторых функций // Тр. ММО. 1959. Т. 8. С. 283-320.

[9] Ван дер Варден В. Л. Алгебра. М.: Наука, 1987.

[10] Кулагин В.А. Об алгебраической независимости значений одного подкласса Е-функций // Диофантовы приближения / ред. П. Л. Ульянов. Сб. статей. Т. II. М.: Изд-во МГУ, 1986. С. 50-63. 